\title{
Management of tracheo-oesophageal fistula in adults
}

\author{
Hyun S. Kim ${ }^{1}$, Danai Khemasuwan ${ }^{1}$, Javier Diaz-Mendoza ${ }^{2}$ and Atul C. Mehta ${ }^{3}$ \\ Affiliations: ${ }^{1}$ Division of Pulmonary and Critical Care, St Elizabeth Medical Center, Boston, MA, USA. \\ ${ }^{2}$ Pulmonary and Critical Care Medicine, Henry Ford Hospital/Wayne State University, Detroit, MI, USA. \\ ${ }^{3}$ Dept of Medicine, Lerner College of Medicine, Respiratory Institute, Cleveland Clinic, Cleveland, OH, USA.
}

Correspondence: Hyun S. Kim, Division of Pulmonary and Critical Care, St Elizabeth Medical Center, 77 Warren St Floor 2, Boston, MA 02135, USA. E-mail: hyun.kimđsteward.org

@ERSpublications

TOF is a complex, challenging condition with a varying degree of acuity, pathogenesis and therapeutic approaches. As such, there is a need for a comprehensive review of methodologies, stenting techniques and promising novel therapeutic methods. https://bit.ly/2ZYIfaZ

Cite this article as: Kim HS, Khemasuwan D, Diaz-Mendoza J, et al. Management of tracheo-oesophageal fistula in adults. Eur Respir Rev 2020; 29: 200094 [https://doi.org/10.1183/16000617.0094-2020].

ABSTRACT Tracheo-oesophageal fistula (TOF) is a pathological connection between the trachea and the oesophagus that is associated with various underlying conditions including malignancies, infections, inhalation injuries and traumatic damage. As the condition spans multiple organ systems with varying aetiologies and acuities, TOF poses unique diagnostic and management challenges to pulmonologists, gastroenterologists and thoracic surgeons alike. Although stents have been a cornerstone in the management of TOF, there exists a large gap in our understanding of their efficacy and precise methodology, making stenting procedure both art and science. TOFs relating to underlying oesophageal or tracheal malignancies require advanced understanding of the airway and digestive tract anatomy, dimensions of the fistula, stent characteristics and types, and the interplay between the oesophageal stent and the airway stent if dual stenting procedure is elected. In this review article, we review the most up-todate data on risk factors, clinical manifestations, diagnostic approaches, management methods and prognosis. Consequently, this article serves to evaluate current therapeutic strategies and the future directions in the areas of 3D-printed stents, over-the-scope clipping systems, tissue matrices and atrial septal closure devices.

\section{Introduction}

Tracheo-oesophageal fistula (TOF) is defined as a pathological connection between trachea and the oesophagus, leading to a spillover of oral and gastric secretions into the respiratory tract [1]. TOF is classified into two main categories: congenital and acquired. Congenital TOF is mainly associated with oesophageal atresia, and was first described by Thomas Gibson in 1696 [2]. Since then, the epidemiology, types of atresia, and repair techniques of congenital TOF have been well described, leading to a significant improvement in its management. In contrast, much less is known about acquired TOF, which mainly affects the adult population.

Acquired TOF is further divided into malignant and benign categories. Each entity makes up approximately half of the acquired cases [3]. The most common cancer associated with malignant TOF is oesophageal cancer, with $>10 \%$ of patients developing the condition during its clinical course [1]. The most common presentations of TOF are respiratory distress, dysphagia and recurrent lung infections; the magnitude of symptoms depend largely on its size and location.

Provenance: Submitted article, peer reviewed

Received: 17 April 2020 | Accepted after revision: 26 May 2020

Copyright $\odot$ ERS 2020. This article is open access and distributed under the terms of the Creative Commons Attribution Non-Commercial Licence 4.0. 
With a median survival of $<3$ months from the time of diagnosis, the management of TOF requires a prompt multidisciplinary approach, including interventional pulmonology, gastroenterology and thoracic surgery [4]. As the respiratory symptoms and complications constitute the majority of initial presentations, the need for awareness of TOF by pulmonologists is heightened. In addition to the traditional approach of using stents, alternative approaches with new devices and technologies have emerged in the management of TOF. We conducted a literature search of peer-reviewed articles in PubMed between 2000 and 2019 using the keywords "acquired tracheoesophageal fistula". This article describes the available data on risk factors, clinical manifestations, diagnostic approaches, traditional and novel management methods and prognosis of acquired TOF.

\section{Aetiology and risk factors}

Benign TOF occurs in the setting of prolonged mechanical ventilation via endotracheal tube or tracheostomy tube; excessive cuff pressure of endotracheal tube or tracheostomy tube; blunt trauma to the chest or the neck; traumatic airway injury; granulomatous mediastinal infections; stent-related injuries; and ingestion of foreign bodies or corrosive products [5]. In the past, the most common cause of acquired benign TOF was from granulomatous mediastinal infection such as tuberculosis. However, with the increased prevalence of intubations and tracheostomies, $\sim 75 \%$ of acquired benign TOFs are deemed iatrogenic [4]. DIDDEE and SHAw [4] estimate that up to $3 \%$ of patients who are mechanically ventilated will form a TOF secondary to cuff-related trauma. As the mechanism of injury stems from pressure necrosis from the cuff, tracheostomies do not reduce the incidence of acquired TOF compared to endotracheal tubes, given the similar mechanism of injury. Other aetiologies of TOF in the setting of mechanical ventilation are traumatic intubation and airway suctioning. Comorbidities such as diabetes, prior airway infections, use of steroids and the presence of nasogastric tubes also increase the risk of TOF formation associated with endotracheal and tracheostomy tubes [6].

Malignant TOF occurs in a setting of cancers arising from the oesophagus, trachea, lungs, larynx and thyroid. In one of the largest case series of malignant TOF involving 207 patients, $77 \%$ were attributed to primary oesophageal cancer and only $16 \%$ to primary lung cancer [7]. Further investigation revealed that the incidence of TOF from oesophageal cancer was $4.3-8.1 \%$ compared to $0.3 \%$ for primary lung cancer [7]. The contrast becomes even more evident with an estimated incidence of $12 \%$ in more locally advanced oesophageal cancer [8]. Given the anatomic proximity of the upper and middle oesophagus to the posterior wall of the trachea and the left mainstem bronchus, tumours originating from the oesophagus can readily invade into the nearby airways. The resulting TOF is usually small in its diameter. However, with the combination of recurrent aspiration injuries, corrosive injuries from gastric acid, pooling of respiratory and gastric secretions, and poor tissue healing from concurrent steroid use, radiation therapy or chemotherapy, the TOF invariably grows rapidly over time, perpetuating a vicious cycle.

There exists a paradox with TOF as the fistula can form due to both cancer progression and cancer treatment. When tumour cells necrose, usually due to chemotherapy or radiation therapy, then the void of the tumour cells between the aerodigestive tracts forms a fistula in their absence. A "clean-edge" airway wall defect can be observed in this setting, and the biopsies are usually negative for any malignancies. CHOI et al. [9] reported that out of 52 patients with TOF and oesophageal cancer, $28.8 \%$ of cases were thought to be related to the treatment of cancer rather than the progression of cancer. Furthermore, BALAzs et al. [8] found that the mean \pm SD latency from the initial radiation therapy to the detection of TOF was $\sim 4.4 \pm 2.98$ months (range 1-13 months, 95\% CI 3.5-5.4 months). This highlights the need for close monitoring of patients undergoing cancer treatment, especially during the first 3-6 months. Similarly, stents have been associated with TOF formation, although they are often the treatment of choice for palliation of TOF. The incidence of oesophageal stent related TOF is estimated to be $4 \%$ with a median latency of 5 months (range $0.4-53$ months) after the placement of the stent [10], again emphasising ongoing monitoring for patients in the post-procedural settings.

\section{Clinical manifestations}

Patients with TOF can have varying clinical manifestations, depending on the rate of its formation, size, location, comorbidities and immunological status of the patient. In a case series involving $>200$ patients by BURT et al. [7], the main symptoms and signs were cough (56\%), aspiration (37\%), fever (25\%), dysphagia (19\%), pneumonia (5\%), haemoptysis (5\%) and chest pain (5\%). Ono's sign (worsening cough with swallowing solid/liquid) was present in $81 \%$ of patients with known TOF, although it is neither sensitive nor specific [11]. The average time for malignant TOF from onset of symptoms to detection is $\sim 7.3 \pm 4.25$ months (range 1-58 months, 95\% CI 6.5-8.1 months). The onset of symptoms in benign cases is more variable, ranging from 5-15 days for traumatic causes to 21-30 days for iatrogenic cuff-related injuries [4]. 
In sedated and ventilated patients, TOF should be suspected if a continued air-leak in the ventilator circuit is detected despite a well-inflated cuff. Other signs, such as abdominal bloating with ongoing ventilation, loss of tidal volume, worsening oxygenation, recurrent pulmonary sepsis and repeated failures to wean can be observed. As TOF is unlikely to heal spontaneously and will eventually lead to respiratory complications and death, a prompt risk stratification and diagnostic efforts are required.

\section{Diagnostic evaluation}

The diagnosis of TOF is made by a combination of thoracic imaging studies and endoscopy (both flexible bronchoscopy and upper endoscopy if possible). An initial investigation of respiratory symptoms with a chest radiograph is a reasonable approach. Depending on the duration of symptoms, early findings of bibasilar infiltrates to more defined basilar consolidative changes can be seen. In addition to aspiration-related changes, the aetiology of TOF may be apparent on initial radiographic evaluation such as a lung mass, overinflated cuff of endotracheal/tracheostomy tube, widened mediastinum from granulomatous disease or a pre-existing oesophageal stent. Although there are no formal guidelines, most experts agree that oesophagography and endoscopy are necessary to diagnose the disease and to perform pre-operative planning. Oesophagography is performed preferentially with barium, given its favourable physiological profile compared to gastrograffin. The latter has been associated with pulmonary oedema and death due to its hypertonic nature [12]. In the presence of TOF, the oral contrast will traverse through the fistula and will be visualised in the airways at oesophagography (figure 1). The contrast-enhanced oesophagogram demonstrates the defect in $\sim 70 \%$ of patients with TOF [13]. The study is not ideal for patients who are not able to swallow the contrast, such as those who are sedated and/or ventilated. In
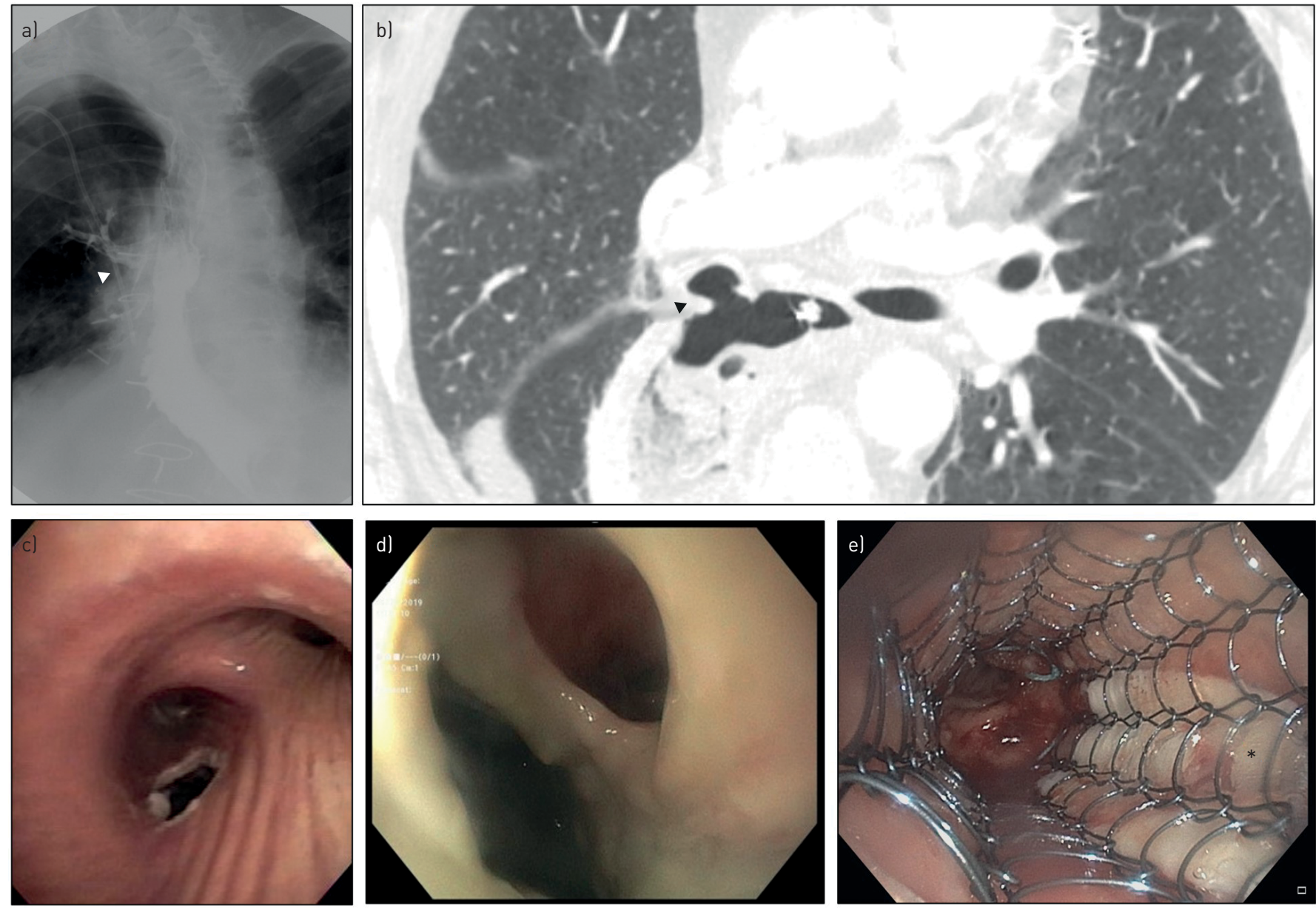

FIGURE 1 A case of malignant tracheo-oesophageal fistula (TOF) after a surgical resection of oesophageal cancer followed by radiation treatment. a) Oesophagogram showing the contrast leak in right upper lobe airways (arrowhead); b) computed tomography scan of the chest showing the defect at the level of right mainstem bronchus (arrowhead); c) bronchoscopic view of the TOF at the posterior wall of proximal right bronchus intermedius; d) oesophageal endoscopic view of the same TOF showing the fistula and mucosal abnormalities; el TOF closure utilising Alloderm $(*)$ and self-expanding metallic stent in the trachea. 
these patients, computed tomography (CT) scan of the chest can be performed to evaluate for signs of fistula, aerodigestive tract anatomy and mediastinal pathology. There are no available data assessing the sensitivity, specificity, negative or positive predictive value of CT scans in settings of known TOF.

Once the thoracic imaging confirms the presence of TOF, the next step in evaluation is to assess the anatomy further via endoscopy and bronchoscopy. Endoscopic visualisation allows for better localisation, measurement and characterisation of the TOF (figures 1 and 2) and can be performed with moderate sedation or general anaesthesia. A direct visualisation can be difficult in the setting of a mucosal inflammation, oedema and gastric debris, which can obscure a small TOF. Gentle manoeuvres with the tip of the endoscope with judicious suctioning can dislodge debris, froth or gastric content, leading to an improved visualisation of the obscured TOF. Similarly, a flexible or a rigid bronchoscope can be used to express purulent material or dislodge spilled gastric contents in the airways to improve the visualisation in the respiratory tract. For patients with poorly visualised fistula due to its size, location or mucosal inflammation, orally administered methylene blue before an endoscopic evaluation can be helpful in the identification of TOF [14]. If the patient is intubated with an endotracheal tube for the procedure, the tip of the endotracheal tube should manoeuvred to allow for a complete visualisation of the airway during the bronchoscopic exam. Endoscopic and/or bronchoscopic biopsies of the lesions should be considered to investigate the underlying aetiology of TOF. The information obtained should be used for careful planning of a palliative procedure.
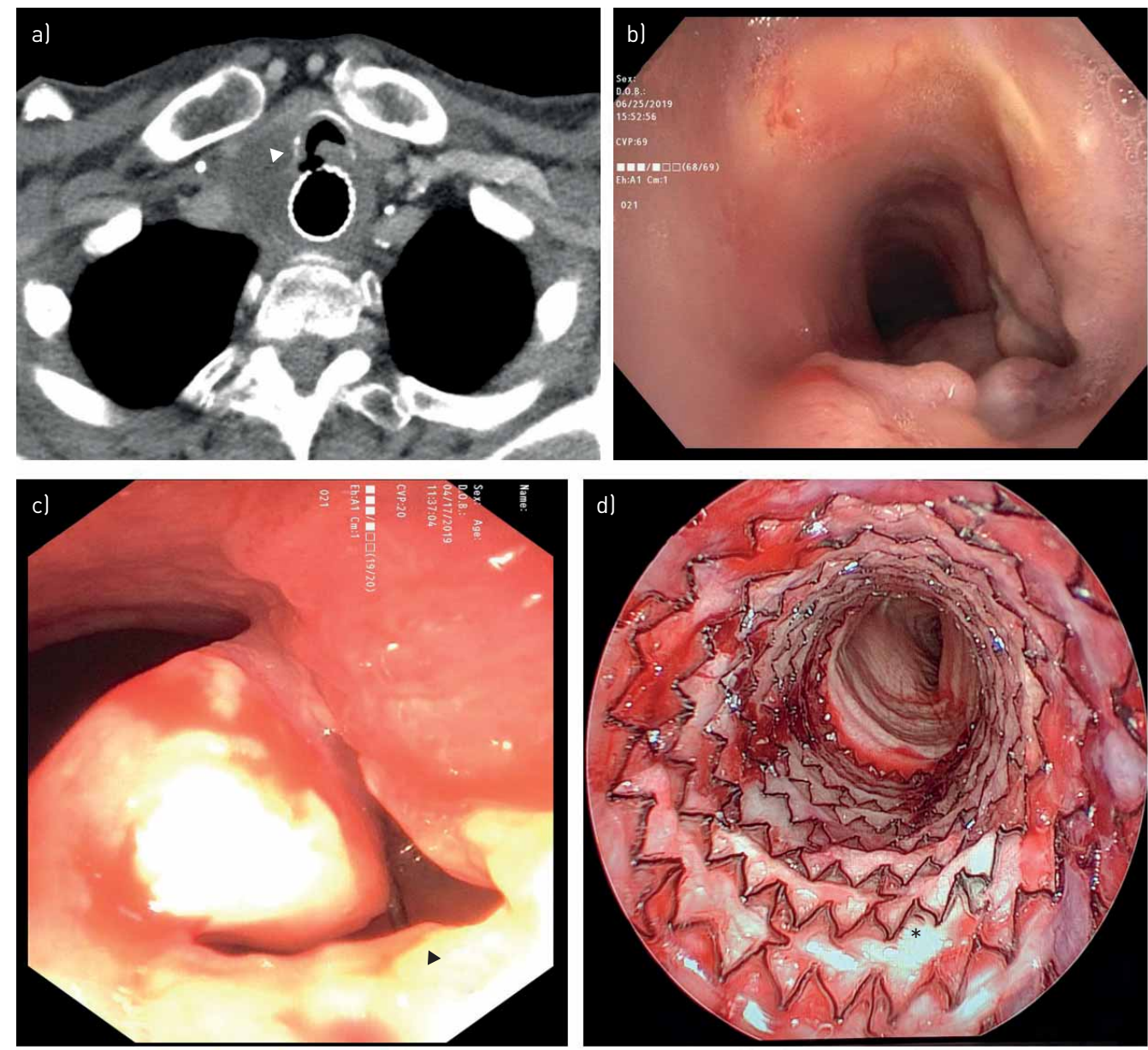

FIGURE 2 A case of malignant tracheo-oesophageal fistula (TOF) from metastatic oesophageal cancer after radiation treatment followed by oesophageal self-expandable metallic stent placement: al computed tomography scan of the chest showing TOF (arrowhead) at the level of proximal end of the oesophageal stent; b) bronchoscopic view showing muscosal abnormality obscuring the view of the fistula at the posterior wall of the trachea. c) A close-up bronchoscopic view of TOF; the oesophageal stent can be seen at the bottom of TOF (arrowhead). d) A case of iatrogenic TOF: closure with Alloderm [*] and covered, self-expandable metallic stent in trachea. 


\section{Location of TOF}

The location of TOF depends on the aetiology and the nature of the inciting injury. For iatrogenic TOFs from cuff-related injuries, the defect will occur in the mid to distal trachea corresponding to the location of the cuff. Most traumatic TOFs are the results of motor vehicle accidents and occur mostly at the level of the carina where the chest wall suffers a forceful crushing injury while striking the steering wheel. The location of TOFs caused by inhalation injuries, aspiration of toxic chemicals and mediastinal infections are less well defined.

The location of malignant TOF largely depends on the location of the primary tumour. From a study by BURT et al. [7] investigating patients with malignant TOFs that were mostly due to oesophageal cancer (77\%), the airway location of the fistula was trachea in 110 (53\%), left main bronchus in 46 (22\%), right main bronchus in $33(16 \%)$, multiple sites in five (2\%) and bronchopleural fistula in 13 (6\%). Another study by BALAzs et al. [8], who looked at patients with oesophageal cancer with concurrent TOF, showed similar trend with the fistula in the trachea/carina in 120 (46\%), right main bronchus in 118 (45\%), left main bronchus in $22(8 \%)$ and distal airways in four $(2 \%)$ patients. The differences in these two studies are probably related to patient population and proportion of patients with oesophageal cancer, but the two studies highlight the consistent relationship between oesophageal cancer and fistula development in the trachea.

\section{Management}

Pre-operative management

The management strategy for TOF should encompass multiple facets of the disease, which includes identifying the underlying aetiology, goals of care and assessing the stability of the overall clinical condition. This is carried out using imaging and endoscopic modalities to conduct pre-operative assessment, and ultimately choosing an endoscopic approach versus a definitive surgical therapy. Prior to undertaking therapeutic interventions, it is crucial to determine and treat the underlying condition implicated in TOF formation.

The general principle of pre-operative management is to treat complications arising from the anatomic deformity, while addressing modifiable risk factors of fistula formation. The most worrisome complication is soiling of the respiratory tract, leading to pneumonitis and ultimately pulmonary sepsis. Acid-suppressive therapy with $\mathrm{H}_{2}$-receptor antagonists or proton-pump inhibitors should be used to decrease the acidity and the volume of gastric acid. Patient positioning with head of bed elevated to $\geqslant 45^{\circ}$, strict limitation of oral intake and frequent oral suctioning are used in conjunction with pharmacological therapy. For ventilated patients, the endotracheal tube can be advanced to position the cuff distal to the fistula to prevent soiling of the respiratory tract. Nasogastric and orogastric tubes should be removed to prevent propagation of the pressure necrosis around the fistula, especially in intubated patients. Placement of gastrostomy tubes for evacuation of residual gastric contents and jejunostomy tubes for enteric feeding can also be considered in appropriate clinical circumstances.

\section{Intra-operative management}

The key principles of intra-operative management parallel those of pre-operative management. It is crucial to consider the aerodigestive tract anatomy and the location of TOF to formulate strategies to minimise spillover of gastric contents into the respiratory tract. As the fistula must be visualised for bronchoscopic treatment, the tip of the endotracheal tube is positioned proximal to the TOF, thus exposing the respiratory tract to gastric contents. Aggressive suctioning via flexible bronchoscope or a suction catheter through the rigid bronchoscope is usually sufficient to clear the gastric spillover in the airways and can result in improved visualisation.

During endoscopy, the most of the concerning complications involving TOF relate to overdistension of the stomach via positive pressure ventilation causing gastric spillover, a unique respiratory complication. Depending on the size and the location of the fistula, the insufflated air from the endoscope can traverse into the airway, leading to increased airway pressures, ineffective ventilation and parenchymal barotrauma. Prompt communication between the endoscopist and the anaesthesiologist is of the utmost importance in preventing complications during the procedure.

\section{Stenting strategy in TOF}

There are two clinical circumstances in which stents can be utilised: bridging benign TOF to definitive surgical therapy, and palliating malignant TOF from symptoms of aspiration, dysphagia, worsening respiratory status and poor nutritional status. In general, benign TOF is more amenable to definitive surgical intervention due to the transient nature of the injury and better nutritional status. However, cardiopulmonary instability may be prohibitive of definite surgical intervention, and may require medical optimisation and extracorporeal device therapy to stabilise the clinical status. In contrast, patients with 
malignant TOF are frequently malnourished and usually are undergoing chemotherapy and/or radiation, making them poor surgical candidates. Minimally invasive endoscopic procedures including stenting are typically preferred in these cases to improve nutritional status while preventing further complications.

The main endoscopic technique to manage TOF is oesophageal and/or airway stenting with the goal to seal the fistula and prevent the spillover to the respiratory tract [15]. Most of the stents are cylindrical in shape, which allow them to exert radial force in the lumen when fully deployed. Thus, oversized stents can stretch the lumen with their expansile radial forces, resulting in an enlargement of the fistula, thus decreasing the possibility of prompt healing. Therefore, stent placement is considered a palliative measure that may increase the quality of life, but may not promote healing of a fistula [15]. In fistulas from malignancies, stent placement is considered the treatment of choice based on poor prognosis with a mean survival expectancy between 1 and 6 weeks [16]. The management algorithm of TOF is shown in figure 3 . The type, quantity and location of stent are very important clinical decisions that pulmonologists and gastroenterologists must make, and the strategy is reviewed here.

\section{Single oesophageal stenting}

Oesophageal stenting is a good option to seal the fistula in the middle and distal sections of the oesophagus, especially in patients without known airway stenosis. In addition, the oesophageal wall is pliable and conforms to the stent. Self-expanding metallic stents (SEMS) are ideal oesophageal stents in the majority of malignant cases, preferable to other available stents such as self-expanding plastic or biodegradable stents. This is due to overall durability, availability and well-established efficacy in a wide variety of malignant oesophageal diseases. When comparing covered SEMS to uncovered SEMS, the covered stents showed increased resistance to tumour ingrowth, but have higher migration rates [17, 18]. For benign oesophageal diseases, self-expanding plastic stents may be considered given their ease of retrieval, as they are used as a bridge to a definitive surgery [19-21]. There is a growing body of evidence preferring the use of fully covered SEMS to treat certain benign oesophageal conditions such as perforations, fistulas or strictures, emphasising the need for an individualised approach to treatment $[22,23]$. For diameter sizing, the use of a balloon catheter in the oesophagus can be used to approximate the appropriate diameter to aid in the stent selection. Known complications from oesophageal stenting are extrinsic airway compression, bleeding, oesophageal perforation, and, paradoxically, formation of new TOFs [24]. For patients at high risk of airway

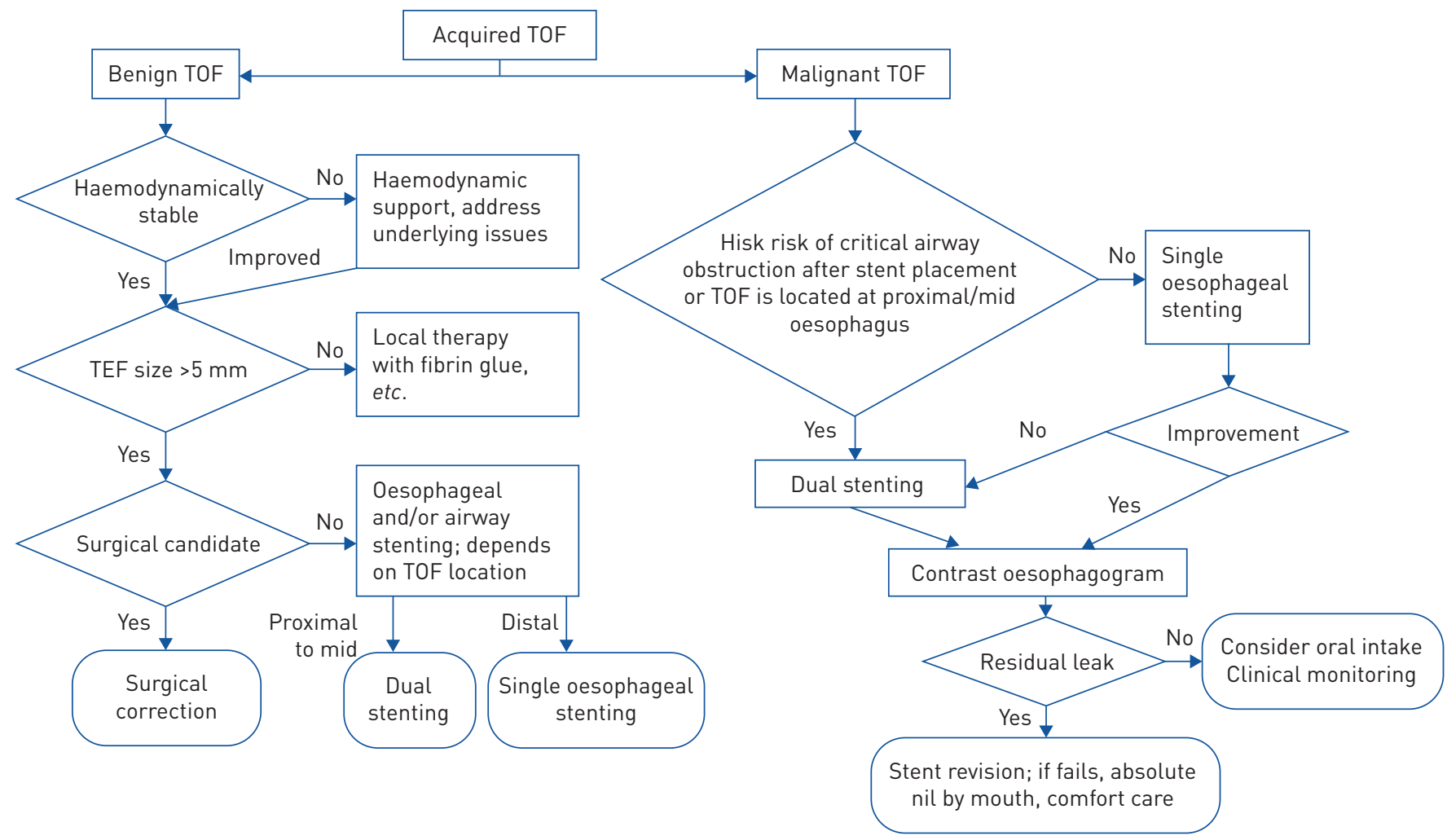

FIGURE 3 Management algorithm for acquired tracheo-oesophageal fistula (TOF). 
obstruction from the extrinsic compression by the oesophageal stent placement, concomitant airway and oesophageal "dual" stenting could be considered under selected circumstances.

\section{Single airway stenting}

There are two main types of airway stents available in the United States: silicone and metallic stents. In patients with TOF, SEMS are generally preferred, owing largely to their ease of deployment and the ability to achieve better apposition to the airway mucosa. They can be placed with a flexible bronchoscope using a guidewire or under direct visualisation, and can be deployed and revised in technically difficult locations compared to silicone stents. As malpositioned stents can obstruct the airways and cause further damage to the fistula or nearby structures, the ability to revise the stent placement is crucial. In addition, SEMS achieve better apposition with the airway wall, which may lower the incidences of migration [25]. However, SEMS have less durability by design, which leads to metal fatigue and stent fracture. This durability is less of a concern in malignant TOF, as these patients rarely survive for more than a few months. Overall, SEMS are favoured in malignant TOFs, evidenced by WANG et al. [26], demonstrating a $71 \%$ complete closure fistula rate.

In contrast, silicone stents are available in different configurations: straight and Y-shaped stents. Silicone stents have studs on the external surfaces, which are designed to prevent migration and reduce mucosal ischaemia [27]. However, these studs may prevent a complete apposition to the airway lumen, thus lessening the sealing effect of the stent. In the management of benign TOFs, silicone stents may be favoured due to better durability compared to SEMS.

The ideal indication for single airway stenting is any pre-existing airway stenosis related to underlying conditions or TOFs in the proximal trachea, where oesophageal stent placement can be technically challenging. This is due to the very proximal oesophageal location of the TOF or an occlusion of the oesophageal lumen from a stenosis or a bulky tumour, making dilation or stenting challenging. The airway stent must be positioned so that the fistula is completely covered, ideally with a covered safety zone of $20 \mathrm{~mm}$ at both ends [28]. Theoretically, this approach provides reassurance against the vertical expansion of the fistula following stent deployment. However, the $20 \mathrm{~mm}$ safety margin may not be achieved due to the location of the fistula.

\section{Dual airway and oesophageal stenting}

Dual stenting may be considered as a first-line intervention in the management of malignant TOF involving the middle to distal trachea [29-32]. However, this practice is controversial, especially in patients with low risk of airway compromise after proper sizing and deployment of oesophageal stents. The dual-stenting approach provides a protective strategy against airway compression by oesophageal stents and their migration into the airways. To further prevent airway compromise, airway stents are always placed first, followed by oesophageal stents. Again, $20 \mathrm{~mm}$ safety margins on the proximal and distal sides of the fistula should be covered by both stents. An additional strategy to ameliorate oesophageal stent migration is placing the upper margin of oesophageal stent higher than the airway stent's upper margin, although its efficacy is unclear. After the deployment of the oesophageal stent, it is important to confirm the position of the airway stent with either bronchoscopic or radiographic examination. One of the main concerns regarding the dual stenting is the risk of enlarging the fistula by the opposing radial forces from airway and oesophageal stents.

Although the stenting strategies of single versus dual stents have been accepted based on anecdotal success and smaller case series, there has not been a head-to-head trial assessing the efficacy of the two approaches. The only available prospective data comes from HERTH et al. [16], who studied the efficacy of dual stenting versus single stenting. Out of 112 patients, 65 (58\%) received a single airway stent, 37 (33\%) received a single esophageal stent and $10(9 \%)$ received dual stents. The unadjusted median survival time was 182, 249 and 245 days, respectively. Other, smaller, retrospective case series are summarised in table 1.

\section{Assessing the efficacy of airway stents}

There are several methods to assess the efficacy of stenting in adequately sealing a TOF. Using a naso/ orogastric tube, methylene blue can be utilised to confirm the proper approximation of stenting and successful closure of the fistula, a concept similar to contrast-enhanced oesophagography. The dye is instilled into the oesophagus and the bronchoscope is used to assess for any leakage of the dye into the airways. If bronchoscopy confirms the lack of spillover, this indicates a successful closure of the fistula. Another method of assessment is using barium swallow test to identify any leakage into to the airway.

For most patients, stent placement helps to prevent aspiration pneumonia. Adequate oral nutrition can be accomplished in a small portion of patients. Oral intake can be resumed with a complete closure of the 


\begin{tabular}{|c|c|c|c|c|c|}
\hline $\begin{array}{l}\text { First author } \\
\text { (year) } \\
\text { [reference] }\end{array}$ & Method & Aetiology & $\begin{array}{l}\text { Location/ } \\
\text { fistula size }\end{array}$ & Sample size $n$ & Outcomes \\
\hline $\mathrm{KE}_{\mathrm{E}}$ (2015) [33] & $\begin{array}{c}\text { Retrospective } \\
\text { case series }\end{array}$ & Undefined & Undefined & $\begin{array}{c}61 \text { (26 tracheal, } \\
35 \text { dual) }\end{array}$ & $\begin{array}{l}\text { Better symptomatic and } \\
\text { radiographic response for } \\
\text { dual stents ( } 96 \% \text { ) versus } \\
\text { tracheal stent (percentage } \\
\text { not specified) }\end{array}$ \\
\hline $\begin{array}{l}\text { HeRth }(2010) \\
\text { [16] }\end{array}$ & $\begin{array}{l}\text { Prospective } \\
\text { case series }\end{array}$ & Malignant & $\begin{array}{c}\text { Trachea, } \\
\text { mainstem } \\
\text { bronchi, size } \\
\text { not reported }\end{array}$ & $\begin{array}{c}112 \text { (65 tracheal, } \\
37 \text { oesophageal, } \\
10 \text { dual) }\end{array}$ & $\begin{array}{l}\text { Better survival time for } \\
\text { dual stents ( } 245 \text { days) } \\
\text { versus tracheal stent } \\
\text { (182 days) }\end{array}$ \\
\hline $\begin{array}{l}\text { FREITAG (1996) } \\
\text { [15] }\end{array}$ & $\begin{array}{c}\text { Retrospective } \\
\text { case series }\end{array}$ & Malignant & $\begin{array}{l}\text { Trachea, } \\
1-4 \mathrm{~cm}\end{array}$ & $\begin{array}{c}30 \text { (12 tracheal, } \\
18 \text { dual) }\end{array}$ & $\begin{array}{l}\text { Better survival time in } \\
\text { dual stents ( } 110 \text { days) } \\
\text { than tracheal stent } \\
\text { (24 days) }\end{array}$ \\
\hline
\end{tabular}

fistula. However, most patients will require nutritional support via gastrostomy, jejunostomy, nasojejunal tube or parenteral nutrition.

\section{Future directions of airway stenting}

One of the major concerns regarding the dual-stenting approach is the paradoxical enlargement of the fistula. Due to its complex anatomy and dynamic motion during the respiratory cycle, an individualised approach to stenting is paramount. With the development of additive manufacturing in biomedical engineering, 3D-printed airway stents have obtained compassionate-use exemption from the United States Food and Drug Administration. Although 3D-printed stents have been used successfully in complex airway diseases such as granulomatosis with polyangiitis and tracheobronchomalacia [34], there is no report of their use in patients with TOF. As the configuration of the airway can be affected substantially by the concomitant placement of oesophageal stents, the exact methodology and procedures for creating 3D-printed individualised stents need to be determined. However, for the same reason, well-designed $3 \mathrm{D}$-printed stents can potentially alleviate pressure necrosis and propagation of fistulae, the major pitfalls of the stenting therapy.

\section{Other therapeutic modalities}

Several alternative therapeutic modalities have been described in the management of TOF, but the majority of these modalities are less well studied, with only case reports to support their use. These modalities are fibrin glue injections, atrial closure devices and micromatrices. Fibrin glue injections have been used to treat small fistulae $(<5 \mathrm{~mm})$. This method has high failure rates in larger fistulae $(>8 \mathrm{~mm})$ due to the rapid dissolution of the coagulative effect, leading to a recanalisation of the fistula [35-37]. The atrial closure device (Amplatzer; AGA Medical, Golden Valley, MN, USA), which was originally designed for transcatheter closure of cardiac defects, has been used to successfully treat TOFs and bronchio-oesophageal fistulae related to nonmalignant aetiologies [38-41]. Significant airway complications have been reported with Amplatzer device use, ranging from airway obstruction from mucostasis and granulation tissue formation to a new TOF from erosive changes relating to the device itself. In addition, unlike airway stent, the Amplatzer decreases the airway cross-sectional area. Thus, this device should not be used in the management of TOF [38]. ACell matrix is a decellularised porcine urinary bladder matrix used to facilitate the natural healing process. This device was used off-label to promote the healing process in patients with benign TOF with a complete closure at 10 days [42]. However, these methods are less often used due to the anecdotal nature and lack of proven efficacy in larger cohorts. Other investigational and alternative therapeutic methods are summarised in table 2.

There are a few reports of bronchoscopic suturing techniques through tracheostomy sites [46], rigid bronchoscopy by using extracorporeal suturing techniques with a knot pusher [47] or a Cor-Knot device [44]. In the report by Mozer et al. [44], the TOF of benign aetiology was closed by suturing the fistula via a rigid oesophageal tube, and securing with a Cor-Knot device. They observed a full post-procedural closure of the fistula on the oesophagogram, and the patient was able to resume full oral intake at 6-month follow-up.

From an endoscopic perspective, a few repair techniques have been described using an oesophageal approach. There are a handful of reports describing successful closure of TOF closure using an 
TABLE 2 Alternative bronchoscopic approaches to stent placement: summary of anecdotal approaches

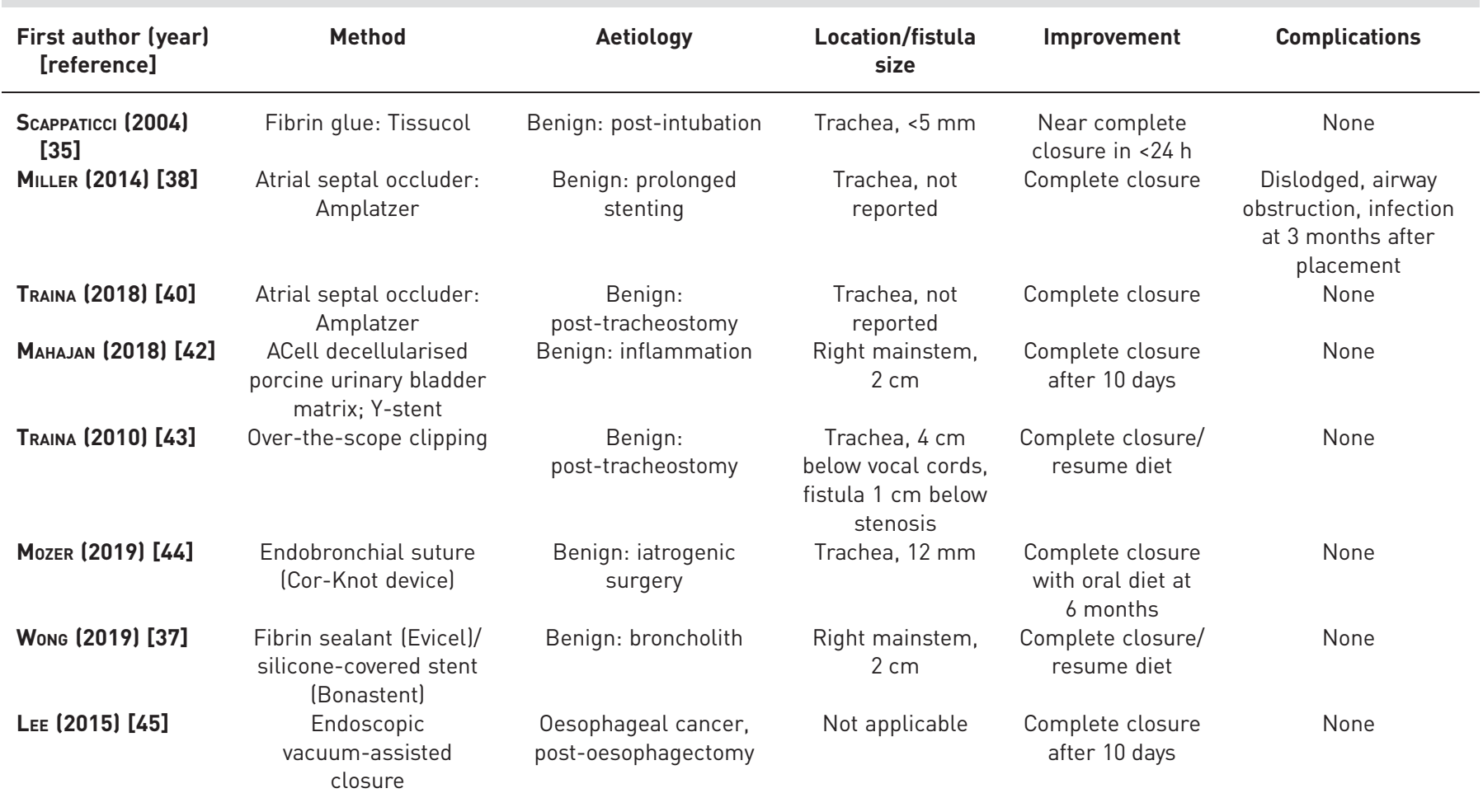

over-the-scope clip system; however, the supporting literature is sparse [43, 48, 49]. Endoluminal vacuum-assisted closure (EVAC) therapy can potentially be used in the repair of TOF. EVAC creates a negative pressure while placing a sponge in the lumen of the fistula. The sponge is connected via a nasogastric tube that continuously removes secretions. This process induces the granulation tissue formation and closure of the fistula [45].

\section{Outcomes}

TOF is a condition that is found in many different conditions: benign, as well as malignant. Thus, it is very difficult to establish concrete outcomes in TOF due to its heterogeneity in affected population. Benign TOFs have more favourable clinical outcomes due to several factors including better nutritional status, fewer comorbidities and feasibility of definitive surgical intervention. In two of the largest recent case series in the surgical literature, perioperative mortality ranged from $0 \%$ to $2.8 \%$ and morbidity ranged from $32 \%$ to $56 \%$ (pneumonias, respiratory failures and fistula recurrences). In a series by MARULLI et al. [50], all 25 patients who underwent surgical intervention for benign TOF survived a median 41 months follow-up.

For malignant TOFs, the available data suggest drastically worse outcomes. Although there was $<0.5 \%$ procedure-related mortality, the mean survival of patients with malignant TOF was only 2.8 months from the time of TOF diagnosis from a large case series by BALAzs et al. [8]. The patients who underwent oesophageal stenting had mean survival of 3.4 months in this study, suggesting potential survival benefits [8]. The clinical efficacy of dual stenting was seen in a retrospective study by FREITAG et al. [15] who found increased survival time compared to single tracheal stenting (110 days versus 24 days). This was supported by a larger prospective study by HerTH et al. [16], with a median survival of 245 days in the dual stent population and 182 days in the single tracheal stent population. Overall, patients who underwent stenting reported a significant improvement in dyspnoea and dysphagia scores, and quality of life measured by the European Organisation for Research and Treatment of Cancer quality-of-life questionnaire (EORTC QLQ-C30), supporting its role in palliative therapy $[16,50]$.

\section{Conclusions}

Due to its heterogeneity in inciting factors and underlying conditions, the diagnosis of TOF is often significantly delayed or undiagnosed. The first step in prompt diagnosis is understanding the pathophysiology of fistula formation between the airways and the oesophagus, and being cognisant of associated conditions. Clinical signs and symptoms can often be helpful, but are very nonspecific, 
requiring careful review of symptoms, ventilatory variables (i.e. loss of tidal volume), frequency of respiratory infections and evolution of chest radiographs. The next step in diagnosis is visualising the fistula via contrast oesophagography or endoscopy/bronchoscopy, which provide crucial pre-procedural planning in addition to the diagnosis. The choice between definitive surgery, palliative stenting, use of investigational methods and conservative approach will depend on combination of patient's condition, underlying aetiology of TOF, goals of care and availability of expertise.

Currently, stenting is the most viable and well-studied intervention for patients with malignant TOF requiring palliative intervention or patients with benign TOF who require stenting to bridge to a definitive surgery. It has shown to improve quality of life in patients with TOF and lessens their symptoms of dyspnoea and dysphagia. The choice of silicone versus metal stent is largely up to the comfort and the experience of the bronchoscopist, as there are advantages and disadvantages to both stents. Single oesophageal stenting is preferred for TOF in the distal oesophagus without known airway compromise. Single tracheal stenting is ideal in very proximal TOF where oesophageal stenting is technically challenging, or with pre-existing airway stenosis. Otherwise, dual stenting may be considered as it provides structural support from both sides of the fistula while creating a seal to prevent spillovers. Furthermore, some studies show improved survival with dual stenting compared to single airway stenting. However, the use of dual stenting is an area of controversy and it depends widely on the clinical practice setting. An individualised approach remains crucial in the management of TOF. Close monitoring of the symptoms and signs of procedural complication is warranted in these highly complex patients to sustain desired clinical outcomes.

Conflict of interest: None declared.

\section{References}

1 Davydov M, Stilidi I, Bokhyan V, et al. Surgical treatment of esophageal carcinoma complicated by fistulas. Eur J Cardiothorac Surg 2001; 20: 405-408.

2 Gibson T. The Anatomy of Humane Bodies Epitomized. 6th Edn London, T. W. for Awnsham and John Churchill, 1703.

3 Reed MF, Douglas JM. Tracheoesophageal fistula. Chest Surg Clin N Am 2003; 13: 271-289.

4 Diddee R, Shaw IH. Acquired tracheo-oesophageal fistula in adults. Contin Educ Anaesth Crit Care Pain 2006; 6: 105-108.

5 Santosham R. Management of acquired benign tracheoesophageal fistulae. Thorac Surg Clin 2018; 28: 385-392.

6 Macchiarini P, Verhoye JP, Chapelier A, et al. Evaluation and outcome of different surgical techniques for postintubation tracheoesophageal fistulas. J Thorac Cardiovasc Surg 2000; 119: 268-276.

7 Burt M, Diehl W, Martini N, et al. Malignant esophagorespiratory fistula: management options and survival. Ann Thorac Surg 1991; 52: 1222-1228.

8 Balazs A, Kupcsulik PK, Galambos Z. Esophagorespiratory fistulas of tumorous origin. Non-operative management of 264 cases in a 20-year period. Eur J Cardiothorac Surg 2008; 34: 1103-1107.

9 Choi MK, Park YH, Im YH, et al. Clinical implications of esophagorespiratory fistulae in patients with esophageal squamous cell carcinoma. Med Oncol 2010; 27: 1234-1238.

10 Bick LB, Song LMWK, Buttar NS, et al. Stent-associated esophagorespiratory fistulas: incidence and risk factors. Gastrointest Endosc 2013; 77: 181-189.

11 Gerzić Z, Rakić S, Randjelović T. Acquired benign esophagorespiratory fistula: report of 16 consecutive cases. Ann Thorac Surg 1990; 50: 724-727.

12 Gore R, Levine M. Textbook of Gastrointestinal Radiology. 3rd edn. Philadelphia, W.B. Saunders Company, 2007.

13 Couraud L, Ballester MJ, Delaisement C. Acquired tracheoesophageal fistula and its management. Semin Thorac Cardiovasc Surg 1996; 8: 392-399.

14 Mathisen DJ, Grillo HC, Wain JC, et al. Management of acquired nonmalignant tracheoesophageal fistula. Ann Thorac Surg 1991; 52: 759-765.

15 Freitag L, Tekolf E, Steveling $\mathrm{H}$, et al. Management of malignant esophagotracheal fistulas with airway stenting and double stenting. Chest 1996; 110: 1155-1160.

16 Herth FJ, Peter S, Baty F, et al. Combined airway and oesophageal stenting in malignant airway - oesophageal fistulas: a prospective study. Eur Respir J 2010; 36: 1370-1374.

17 Sarper A, Oz N, Cihangir C, et al. The efficacy of self-expanding metal stents for palliation of malignant esophageal strictures and fistulas. Eur J Cardiothorac Surg 2003; 23: 794-798.

18 Sharma P, Kozarek R, Practice Parameters Committee of American College of Gastroenterology. Role of esophageal stents in benign and malignant diseases. Am J Gastroenterol 2010; 105: 258-257.

19 Verschuur EML, Repici A, Kuipers EJ, et al. New design esophageal stents for the palliation of dysphagia from esophageal or gastric cardia cancer: a randomized trial. Am J Gastroenterol 2008; 103: 304-312.

20 Ham YH, Kim GH. Plastic and biodegradable stents for complex and refractory benign esophageal strictures. Clin Endosc 2014; 47: 295-300.

21 Kang Y. A review of self-expanding esophageal stents for the palliation therapy of inoperable esophageal malignancies. Biomed Res Int 2019; 2019: 9265017.

22 Buscaglia JM, Ho S, Sethi A, et al. Fully covered self-expandable metal stents for benign esophageal disease: a multicenter retrospective case series of 31 patients. Gastrointest Endosc 2011; 74: 207-211.

23 Eloubeidi MA, Talreja JP, Lopes TL, et al. Success and complications associated with placement of fully covered removable self-expandable metal stents for benign esophageal diseases (with videos). Gastrointest Endosc 2011; 73: 673-681. 
24 Wang MQ, Sze DY, Wang ZP, et al. Delayed complications after esophageal stent placement for treatment of malignant esophageal obstructions and esophagorespiratory fistulas. J Vasc Interv Radiol 2001; 12: 465-474.

25 Avasarala SK, Freitag L, Mehta AC. Metallic endobronchial stents: a contemporary resurrection. Chest 2019; 155: $1246-1259$.

26 Wang $\mathrm{H}$, Tao M, Zhang N, et al. Airway covered metallic stent based on different fistula location and size in malignant tracheoesophageal fistula. Am J Med Sci 2015; 350: 364-368.

27 Flannery A, Daneshvar C, Dutau H, et al. The art of rigid bronchoscopy and airway stenting. Clin Chest Med 2018; 39: 149-167.

28 Hürtgen M, Herber SC. Treatment of malignant tracheoesophageal fistula. Thorac Surg Clin 2014; 24: 117-127.

29 Matsumoto K, Yamasaki N, Tsuchiya T, et al. Double stenting with silicone and metallic stents for malignant airway stenosis. Surg Today 2017; 47: 1027-1035.

30 Machuzak MS, Santacruz JF, Jaber W, et al. Malignant tracheal-mediastinal-parenchymal-pleural fistula after chemoradiation plus bevacizumab: management with a Y-silicone stent inside a metallic covered stent. J Bronchology Interv Pulmonol 2015; 22: 85-89.

31 Zhou C, Hu Y, Xiao Y, et al. Current treatment of tracheoesophageal fistula. Ther Adv Respir Dis 2017; 11: $173-180$.

32 Nomori H, Horio H, Imazu Y, et al. Double stenting for esophageal and tracheobronchial stenoses. Ann Thorac Surg 2000; 70: 1803-1807.

33 Ke M, Wu X, Zeng G. The treatment strategy for tracheoesophageal fistula. J Thoracic Dis 2015; 7: Suppl. 4, S389-S397.

34 Alraiyes AH, Avasarala SK, Machuzak MS, et al. 3D printing for airway disease. AME Med J 2019; 4: 14.

35 Scappaticci E, Ardissone F, Baldi S, et al. Closure of an iatrogenic tracheo-esophageal fistula with bronchoscopic gluing in a mechanically ventilated adult patient. Ann Thorac Surg 2004; 77: 328-329.

36 Sharma M, Somani P, Sunkara T, et al. Trans-tracheal cyanoacrylate glue injection for the management of malignant tracheoesophageal fistula. Am J Gastroenterol 2018; 113: 800.

37 Wong A-K, McDonald A, Jones B, et al. Patch-and-glue technique in bronchoesophageal fistula repair and broncholith removal with stent and fibrin glue. Chest 2019; 156: A1191.

38 Miller PE, Arias S, Lee H, et al. Complications associated with the use of the amplatzer device for the management of tracheoesophageal fistula. Ann Am Thorac Soc 2014; 11: 1507-1509.

39 Marwah V, Rajput AK, Madan H, et al. Closure of chronicbronchopleural fistula using atrial septal occluder device. J Bronchology Interv Pulmonol 2014; 21: 82-84.

40 Traina M, Amata M, De Monte L, et al. Chronic tracheoesophageal fistula successfully treated using Amplatzer septal occluder. Endoscopy 2018; 50: 1236-1237.

41 Fruchter O, Kramer MR, Dagan T, et al. Endobronchial closure of bronchopleural fistulae using amplatzer devices: our experience and literature review. Chest 2011; 139: 682-687.

42 Mahajan AK, Newkirk M, Rosner C, et al. Successful endobronchial treatment of a non-healing tracheoesophageal fistula from a previous histoplasmosis capsulatum infection using decellularized porcine urinary bladder matrix. J Surg Case Rep 2018; 2018: rjy187.

43 Traina M, Curcio G, Tarantino I, et al. New endoscopic over-the-scope clip system for closure of a chronic tracheoesophageal fistula. Endoscopy 2010; 42: Suppl. 2, E54-E55.

44 Mozer AB, Michel E, Gillespie C, et al. Bronchoendoscopic repair of tracheoesophageal fistula. Am J Respir Crit Care Med 2019; 200: 774-775.

45 Lee HJ, Lee H. Endoscopic vacuum-assisted closure with sponge for esophagotracheal fistula after esophagectomy. Surg Laparosc Endosc Percutan Tech 2015; 25: e76-e77.

46 Caronia FP, Reginelli A, Santini M, et al. Trans-tracheostomy repair of tracheo-esophageal fistula under endoscopic view in a 75-year-old woman. J Thorac Dis 2017; 9: E176-E179.

47 Galluccio G. Endoscopic treatment of tracheo-oesophageal fistulae: an innovative procedure. Multimed Man Cardiothorac Surg 2016; 201: mmw015.

48 Armellini E, Crinò SF, Orsello M, et al. New endoscopic over-the-scope clip system for treatment of a chronic post-surgical tracheoesophageal fistula. Endoscopy 2015; 47: Suppl. 1, E437-E438.

49 So BJ, Adler DG. Closure of a chronic, non-healing tracheoesophageal fistula with a new over-the-scope clip. ACG Case Rep J 2014; 2: 18-20.

50 Marulli G, Loizzi M, Cardillo G, et al. Early and late outcome after surgical treatment of acquired non-malignant tracheo-esophageal fistulae. Eur J Cardiothorac Surg 2013; 43: e155-e161. 